\title{
Menstrual Issues in Adolescents with Physical and Developmental Disabilities
}

\author{
ELISABETH H. QUINT \\ Department of Obstetrics and Gynecology, Division of Gynecology, University \\ of Michigan Health System, Ann Arbor, Michigan, USA
}

\begin{abstract}
Puberty and menstruation are difficult issues for teens with disabilities and for their families as well. Irregular bleeding, mood swings, and problems with hygiene often complicate the delicate balance in the lives of these adolescents. The care provider is asked to help with the pubertal transition and the issues surrounding menstruation and reproductive health. This review will concentrate on the issues concerning menstruation that need special focus in teens with developmental disability (DD). The impact of the menstrual cycle on these teenagers, including hygiene issues, menstrual irregularities due to specific clinical circumstances, and treatment dilemmas associated with the use of hormonal medication will be discussed for this special group of teens.
\end{abstract}

Key words: menstruation; adolescents; developmental disabilities; physical disabilities; menstrual hygiene

\section{Introduction}

Pubertal development and the subsequent onset of menses are the beginning of a significant change for many adolescents. This may even be more for a teen with developmental disability (DD), for whom life itself is challenging, and mental and physical health is often in a delicate balance. The changes attendant on puberty can cause this balance to shift significantly. The care provider for these adolescents will be called upon to help manage this transition time and guide the teen and her family through this time by means of education, understanding, and good medical care.

This review will concentrate on issues concerning menstruation and reproduction, areas that need special focus in teens with DD. Menstrual cycle problems related to the disabilities, including clinical circumstances like medications and issues related to hygiene, will be addressed. Treatment dilemmas associated with the use of hormonal medications specific to teens with disabilities will also be discussed. Data are sparse on many issues, and research in women with developmental disabilities is often retrospective because of circumstances requiring informed consent. Many recommendations are therefore extrapolated from the literature on the general population.

Address for correspondence: Elisabeth H. Quint, M.D., University of Michigan Health System, Department of Obstetrics and Gynecology, L4000 Women's Hospital, 1500 E. Medical Center Drive, Ann Arbor, MI 48109-0276. Voice: 734-764-8429; fax: 734-647-9727.

equint@med.umich.edu

\section{Issues Specific to the Menstrual Cycles of Teens with DD}

\section{Epidemiology}

Studies from the United States suggest a lowering in the age of onset of puberty over the past 40 years of between 0.5 and 1.0 years, with black girls maturing 0.5 to 1 year earlier than white girls. There has been a smaller decrease in the mean age at menarche on the order of 0.2 years. $^{1}$ The most recent results from the NHANES (National Health and Nutrition Examination Survey) study suggest that the mean age of menarche declined by 0.9 year overall in women born before 1920 compared to women born in 1980-84; the declines in the mean age at menarche ranged from 0.7 to 1.4 years, depending on the race/ethnic group. ${ }^{2}$ There is little information on the epidemiology of menstrual cycles in women with developmental disabilities. One study looking at 35 women with autism spectrum conditions found an average delayed age of menarche by 8 months (13 years and 4 months as opposed to 12 and 7 months in a control group) They also found 4 girls with extreme late onset of puberty (17-20 years). ${ }^{3}$ Looking at a large group of children with cerebral palsy, puberty began earlier, but ended later in white children with CP, compared with white children in the general population. In addition, menarche occurred at age 14 in white girls with $\mathrm{CP}^{4}{ }^{4} \mathrm{FSH}$ and $\mathrm{LH}$ responses to $\mathrm{GnRH}$ stimulation in teenagers with and without developmental disabilities showed an impaired response of the FSH-secreting pituitary cells in initial pubertal stages. This difference disappeared during further sexual development. ${ }^{5}$ 
Although one study found that in women with Down syndrome with regular cycles, ovulatory events were less frequent and often characterized by luteal phase defects,${ }^{6}$ another study could not confirm that. ${ }^{7}$ Overall, there are no clear data to suggest that the onset of menarche or the cycles of women with developmental disabilities are very different due to the disability, except in some specific conditions, like autism. There are, however, several other common factors that may contribute to menstrual irregularities.

\section{Common Factors That Contribute to Menstrual Irregularities in Women with $D D$}

Women with epilepsy have an increased incidence of reproductive endocrine disorders, including irregular menstrual cycles, anovulatory cycles, amenorrhea. and oligomenorrhea. ${ }^{8}$ Polycystic ovary syndrome occurs in 10-20 percent of women with epilepsy compared to 5-6 percent in the general population. Women on valproic acid may have an even higher incidence of up to $60 \% .^{9-11}$ Many anticonvulsants, including carbamazepine, felbamate, fenytoin and phenobarbitol, increase the activity of the cytochrome P450 hepatic micrososmal oxidative enzymes. This results in more rapid clearance of steroid hormones and may lead to menstrual irregularities. Antipsychotics and metoclopramide can cause hyperprolactinemia, which can lead to abnormal bleeding and ultimately to amenorrhea due to hypoestrogenemia with concerns about bone loss. ${ }^{12}$

Thyroid disease, which can lead to subsequent disturbance of the cycle, is more prevalent in women with Down syndrome. ${ }^{13}$ Teens with swallowing problems or feeding tubes may have such a low weight that they experience hypothalamic oligo- or amenorrhea.

\section{Menstrual Hygiene}

Issues surrounding menstrual hygiene are a common point of discussion with teens with DD and their families. ${ }^{14}$ Once menarche occurs, girls with DD may have physical challenges that may make menstrual hygiene difficult, or they may be unable to deal with menstrual pads. The teen may remove the pads in inappropriate places on account of a heightened sensory awareness of discomfort from the pads. Some teens cannot physically change their own pads, which may interfere with their ability to be independent; this may be seen in teens with CP or spina bifida. Other girls may have difficulty communicating about the menstrual cycle. Problems may arise if caregivers at school or at home are unable or unwilling to help with hygiene, so that the teens may end up staying home from school. Some of these issues can be remedied with patient or caregiver education, focusing on the normalcy of periods in all women, explaining that this is not "painful or bad blood," and providing help to the family and school caregivers with the management of pads. This education will include the adolescent (most teens that are toilet-trained can be taught how to deal with pads) and her family or the school caregivers, making sure that all issues are addressed.

\section{Amenorrhea}

Although having no periods may appear to be a good thing for the teen and her family, amenorrhea may be an indication of anovulation, with its potential for endometrial hyperplasia if left untreated. Low weight or hyperprolactinemia can cause low estrogen levels and subsequent amenorrhea. Secondary amenorrhea during the years of adolescence may play a role in the development of osteoporosis or osteopenia in later years. ${ }^{15}$ A diagnosis for the cause of amenorrhea needs to be made, and the issues discussed with the patient and her family, so that an appropriate plan can be devised.

\section{Request for Birth Control: Assessment of Sexuality and Education}

The call for cessation or management of menses may also be combined with an underlying request for birth control. If the request comes from the teenager, the clinician should plan to spend some private time with her and try to assess whether she could consent to a sexual relationship. The clinician should investigate the adolescent's level of sexual education. Simple terms and repetition of topics should be used when educating adolescents with special needs. Visual aids, pictures, signs, symbols, and anatomically correct dolls or models are very useful to assess knowledge and help with understanding.

If the request for menstrual suppression comes from the caregivers, assess the adolescent's safety at home and at school. Always address the risk for abuse, which is thought to be increased in teens with disabilities. ${ }^{16}$

\section{Cyclical Behavioral Changes}

Cyclical behavioral changes are a common issue for women with developmental disabilities $(16 \%) .{ }^{17}$ The cyclical behavior in women with DD may be very different from what is typically thought of as PMS in adult women; it may include temper tantrums, crying spells, autistic behavior, self-abusive behavior, or seizures. Menstrual calendars in combination with symptom calendars are used to make the diagnosis of menstrual-related mood and behavioral symptoms, as well as to assess treatment success. The 
diagnostic criteria for PMS as described in the ACOG Committee Opinion on PMS indicate that one of the described symptoms in the affective and somatic categories should be present in the 4 days prior to menses and not reoccur until in the 12 days prior to the next cycle in three consecutive cycles. ${ }^{18}$ For teens with DD, help is usually needed to document symptoms: parents and teachers need to keep daily records of the most bothersome symptoms for 3 months. In that way, true menstrual cyclicity can be documented and other behavior patterns can be investigated. Dysmenorrhea is very common in teenagers $(10-45 \%),{ }^{19}$ and teens who cannot communicate their discomfort or pain may manifest changes in behavior. Once cyclical behaviors are documented, non-steroidal anti-inflammatory drugs (NSAIDs) should be the first-line treatment for suspected dysmenorrhea; NSAIDs should be given in appropriate doses starting on the first day of behavioral changes. This was found to be successful in two-thirds of adult patients with DD. ${ }^{17}$ If that is unsuccessful, menstrual manipulation can be attempted with oral contraceptives or progestins. An oral contraceptive with drospirenone may be helpful. ${ }^{20}$ Menstrual suppression with depot medroxyprogesterone has also been used, although data to support behavioral benefits are sparse. Success of these treatments in women with disabilities has not been well studied.

Selective serotonin reuptake inhibitors (SSRIs) have been used extensively to manage severe PMS and PMDD in the general population and have been found to be very helpful. ${ }^{21}$ However, this class of medication has not been studied for this indication in women with DD. In view of recent warnings of suicides in teens on certain SSRIs, as well as the possibility of interaction with the many medications that teens with DD take, caution and a consultation with a neurologist and/or psychiatrist are recommended.

\section{Diagnosis and Work-Up}

If a teen with DD and her family come to discuss menstruation, the scope of the issues needs to be determined. Is there truly abnormal bleeding, severe dysmenorrhea or anemia, or is there a request for hygiene or a concern about sexual activity or abuse? Do the cycles affect the life of the teenager and her caregivers negatively? A bleeding calendar may be helpful. Irregular anovulatory bleeding due to the immature hypothalamic-pituitary-ovarian axis is very common during the first two post menarchal years and may persist for up to 5 years. ${ }^{22}$ Reassurance is often all that is needed. If the bleeding is excessive or leads to hospi- talization, a thorough work-up is indicated, including hemoglobin, thyroid function testing, prolactin screening, and a search for bleeding disorders.

Ultrasounds or pelvic exams are rarely indicated in adolescents with DD and menstrual abnormalities, as structural lesions as a cause for abnormal bleeding in teens are unusual. The sexually active teen needs to be screened appropriately by cytologic study of the cervix and for STI per screening guidelines for all adolescents.

\section{Treatment}

The decision to treat the patient's menstruation is based on an individual assessment of the menstrual cycle, the tolerance of the patient of her periods and the products used, as well as the impact of the cycles on her daily activities. Routine requests for suppression of puberty and menstruation without any particular identified problem should be resisted. ${ }^{23}$ Once the decision is made to treat, treatment goals should be set with the teen and her family. Goals could be to: decrease flow, relieve pain or symptoms, provide contraception, or obtain amenorrhea. Continued documentation of the cycles by the family is very helpful to assess the efficacy of treatment.

Before prescribing any hormonal treatment, a clinician needs to realize that once interventions are started, they are often continued indefinitely. Periodic evaluation of continuation of treatment should be part of the long-term goal.

The use of non-steroidal anti-inflammatory drugs (NSAIDs) lessens dysmenorrhea and can decrease menstrual flow by up to $20 \% .^{24}$ The dose needs to be clearly prescribed, with adequate dosing intervals, not on an as-needed basis. NSAIDs should be used throughout the entire menstrual period. After 3 months, the effect of this course of treatment can be assessed and if the desired outcome is not reached, hormones are the next logical step.

\section{Combined Contraceptives}

If regularity or decrease in flow and cramping is desired, low-dose combined contraceptives are a good choice. Consistent pill intake is often an issue for adolescents, and should be monitored closely by the parents of girls with DD. If swallowing is difficult, the pill can be taken with a favorite food; a chewable oral contraceptive is available, or a transdermal patch can be used. Extended cycling is now widely used to achieve amenorrhea (up to $90 \%$ after the first few months) and early studies show no concerns about safety. ${ }^{25}$ Troublesome 
spotting can be treated by taking two pills for several days or taking periodic 4-7 day pill-free intervals to allow for a withdrawal bleed. ${ }^{26}$ Contraindications to estrogen/progestin combination oral contraceptives are the same as in the general population, but there are some specific concerns related to girls with DD. Deep venous thrombosis (DVT) may be a concern, in the context of decreased mobility for some girls with DD. DVT is a disease with many risk factors, subdivided into three groups: hypercoagulability, venous stasis, or endothelial damage. There are few studies that address this issue for women with DD. One small retrospective study found that no DVT was recorded in 57 institutionalized residents during 18 years (but no testing for asymptomatic DVT was performed). ${ }^{27}$ One case report describes a tetraparesic woman on OCP who sustained a DVT. ${ }^{28}$ Most of the studies on DVT and immobilization have been related to air travel, and suggest there may be other risk factors in this situation, such as hyperbaric hypoxia. ${ }^{29}$ However, we know that prolonged sitting does increase venous stasis and hence theoretically the risk for DVT; it seems prudent to be cautious with combined contraceptives. Recommendations include using the lowest dose estrogen possible and avoiding third-generation progestins, as they have been reported to increase the risk of DVT twofold compared with second-generation OCPs. ${ }^{30} \mathrm{~A}$ careful family history to assess for DVT/pulmonary emboli is recommended ${ }^{31}$ and screening for the common inherited thrombofilia factor $\mathrm{V}$ Leiden should be considered if a positive family history is obtained.

The transdermal patch can be used in women with problems swallowing or forgetfulness, but the higher estrogen levels have been reported to be associated with a twofold increase in DVT compared to a norgestimatecontaining oral contraceptive. ${ }^{32}$ The patch can cause skin irritation in some. Occasionally girls with DD will find the presence of the patch bothersome, and may attempt to remove it. Placing the patch on the lower part of the back may solve that problem.

The vaginal ring is usually not used in this population because of issues concerning placement and removal, which would need to be done by caregivers.

Some preliminary data on the 20-mcg ethinyl estradiol pills suggest possible concern about bone mineral density and bone accretion. ${ }^{33}$

Patients on enzyme-inducing antiepileptic drugs (AEDs) may have decreased serum levels of estrogen and progestin, potentially leading to abnormal bleeding patterns and decreased contraceptive efficacy. ${ }^{34}$

\section{Progestin-Only Methods Oral Progestins}

If a teen has clearly anovulatory cycles, oral progestins like medroxyprogesterone, norethindrone, or micronized progesterone, can be used for 10 days out of the month. This complicated dosing regimen, and the fact that it does not protect against pregnancy, make it less desirable than oral contraceptives, but with good supervision this can be used to regulate the cycles. If amenorrhea is the goal, continuous oral progestins can be used. Side effects include breakthrough bleeding or mood disturbances, as well as an increased appetite with megestrol acetate.

\section{Depot Medroxyprogesterone Acetate}

If complete cessation of menstruation is the goal, depot medroxyprogesterone acetate (DMPA) $150 \mathrm{mg}$ given intramuscularly every 12 weeks (every 10 weeks in women on enzyme-inducing AEDs) has been used successfully, with amenorrhea rates approaching $70 \%$ over time. Subcutaneous medroxyprogesterone acetate has similar rates of amenorrhea ( $55 \%$ at 1 year). ${ }^{35}$ The main concerns with DMPA are weight gain and its impact on bone mineral density.

Both subcutaneous and intramuscular depot medroxyprogesterone have been associated with weight gain. This weight gain shows large variations in several studies, but an average weight gain of 4.5 and $5.8 \mathrm{~kg}$ in three years was found in a comparison study. ${ }^{36}$ Weight gain is of particular concern in this patient population, as the added body weight may make mobility and transfers more difficult. Previous research has shown a significant relationship between increased body weight on DMPA and initial weight status, leading to higher gains in the already obese. ${ }^{37}$

Longitudinal studies have demonstrated consistent loss in bone mass of $1-2 \%$ per year in DMPA users, regardless of age. In adolescents, a $1-2 \%$ bone loss with use of DMPA is dramatically different from the increase in bone density (up to $9 \%$ annually) that is expected during adolescence without the use of DMPA. ${ }^{38}$ Therefore, use of DMPA in the adolescent may potentially compromise adult peak bone mass, with the potential for earlier osteoporosis and subsequent fractures. There are now data suggesting that most bone mass returns after stopping the injections. ${ }^{33}$ Whether the fracture rate is affected has not yet been adequately researched. ${ }^{39}$

Women with DD may have coexisting physical disorders limiting mobility, which may affect bone density. Significant decreases in bone mineral density scores have been found in adults and children with physical disabilities. ${ }^{40,41}$ One large retrospective study using 
administrative data of 6,773 women and teens with DD showed an increased fracture risk of 2.4 among 340 users of DMPA compared to nonusers, and a RR for fractures of 1.9 among 1,909 users of AEDs. ${ }^{42}$ One chart review in a primary care practice found more reported fractures in adult women with DD then in those without DD; these occurred at an average age of $41 .{ }^{43}$

The issue of wheelchair use and the role that this plays in bone mineral density has not been extensively researched. One study from Europe suggested decreased bone density in wheelchair-bound patients; however, the sample studied was small. ${ }^{44}$ The importance of dietary intake or supplementation of vitamin $\mathrm{D}$ and calcium needs to be emphasized, as an optimal peak bone mass may not be reached if intake is inadequate.

\section{Implantable Progestin}

The implantable progesterone rod, Implanon, has not been studied in women with DD. Data suggest an amenorrhea rate of $33 \%$, but it causes significant unscheduled bleeding, which may make it less desirable in this population. ${ }^{45}$ Insertion may require sedation or anesthesia and the implant is not recommended for women on enzyme-inducing AEDs.

\section{Levonorgestrel Intrauterine Device}

The levonorgestrel-releasing intrauterine system (LNG-IUS) causes a decrease in menstrual flow that may become an alternative treatment for heavy cycles in women with DD who have a normal uterine cavity. This use of the device is off-label. With the LNG-IUS, rates of amenorrhea are significant and side effects are limited. ${ }^{46}$ In girls with DD, insertion may need to be done under anesthesia, due to nulligravid status, unpredictable cooperation, as well as the potential for a small uterus. One study reported the insertion as difficult in $14.9 \%$ of nulliparous women aged 18-25, compared to $15.4 \%$ in a largely multiparous population, and the IUD could not be placed in two cases $(2.1 \%){ }^{47}$

\section{Surgical Alternatives}

Usually hormonal treatment is adequate to address menstrual issues in women with DD, but surgical measures, like endometrial ablation and hysterectomy may be requested by the family, and need to be addressed. ${ }^{48}$ Both these measures would render the patient sterile, which has ethical and legal implications. ${ }^{49}$ This is very difficult decision, so in these rare circumstances, the clinician should strongly consider consulting an ethics committee and be aware of state laws.

\section{Conclusion}

The management of menstrual issues in adolescents with developmental disabilities should be guided by the same principles that apply to the general teen population. The medical careproviders should work with the patients, parents, and caregivers to find an acceptable course of action. Clinicians should always be concerned about the vulnerability of this population. If the cycles are limiting the patient's ability to participate in her regular activities and are clearly affecting her life, treatment can be initiated with periodic evaluation of the need for continuation. There is a paucity of data on treatment options specific for women with DD. The general concerns are outlined in this monograph, although many recommendations are based primarily on research in the general teen population.

\section{Conflicts of Interest}

The author declares no conflicts of interest.

\section{References}

1. Kaplowitz, P. 2006. Pubertal development in girls: secular trends. Curr. Opinion Obstet. Gynecol. 18: 487-491.

2. McDowell, M.A., D.J. Brody \& J.P. Hughes. 2007. Has age at menarche changed? Results from the National Health and Nutrition Examination Survey (NHANES) 1999-2004. J. Adolesc. Health 40: 227-231.

3. Knickmeyer, R.C., S. Wheelwright, R. Hoekstra \& S. Baron-Cohen. 2006. Age of menarche in females with autism spectrum conditions. Devel. Med. Child Neurol. 48: $1007-1008$.

4. Worley, G., G.M. Houlihan, M.E. Herman-Giddens, et al. 2002. Secondary sexual characteristics in children with cerebral palsy and moderate to severe motor impairment: a cross-sectional survey. Pediatrics 110: 897-902.

5. Gento, R.M., M. Ciampelli, C. Proto, et al. 2001. Neuroendocrine features of pubertal development in females with mental retardation. Gynecol. Endocrinol. 15: 178183.

6. Cento, R.M., L. Ragusa, C. Proto, et al. 1996. Basal body temperature curves and endocrine pattern of menstrual cycles in Down syndrome. Gynecol. Endocrinol. 10: 133137.

7. Scola, P.S. \& S.M. Pueschel. 1992. Menstrual cycles and basal body temperature curves in women with Down syndrome. Obstet. Gynecol. 79: 91-94.

8. Bauer, J., J.L. Isojarvi, A.G. Herzog, et al. 2002. Reproductive dysfunction in women with epilepsy: recommendations for evaluation and treatment. J. Neurol. Neurosurg. Psych. 73: 121-125.

9. Bilo, L., R. Meo, C. NAPPI, et al. 1988. Reproductive endocrine disorders in women with primary generalized epilepsy. Epilepsia 29: 612-619. 
10. Herzog, A.G. \& S.C. Schachter. 2001. Valproate and the polycystic ovarian syndrome: final thoughts. Epilepsia 42: 311-315.

11. Isojarvi, J.I.T., T.J. Latikainen, A.J. Pakarinen, et al. 1993. Polycystic ovaries and hyperandrogenism in women taking valproate for epilepsy. N. Engl. J. Med. 329: 1383-1388.

12. Wieck, A. \& P.M. Haddad. 2003. Antipsychotic-induced hyperprolactinaemia in women: pathophysiology, severity and consequences: selective literature review. Br. J. Psych. 182: 199-204.

13. Cohen, W.I. 2006. Current dilemmas in Down syndrome clinical care: celiac disease, thyroid disorders, and atlantoaxial instability. Am. J. Med. Genet. Semin. Med. Genet. 142: $141-148$.

14. Dizon, G.D., L.M. Allen \& M.P. Ornstein. 2005. Menstrual and contraceptive issues among young women with developmental disability: a retrospective review of cases of the Hospital for Sick Children, Toronto. J. Ped. Adolescent. Gynecol. 18: 157-162.

15. Csermely, T., L. HalvaX, M. Vizer et al. 2007. Relationship between adolescent amenorrhea and climacteric osteoporosis. Maturitas 56: 368-374.

16. Murphy, N.A. \& E.R. Elias. 2006. Sexuality of children and adolescents with developmental disabilities. Pediatrics 118: $398-403$.

17. Quint, E.H., T.E. Elkins, C.A. Sorg \& S. KoPE. 1999. The treatment of cyclical behavioral changes in women with mental disabilities. J. Ped. Adolesc. Gynecol. 12: 139-142.

18. American College of Obstetrics and Gynecology. 2000. ACOG practice Bulletin: Premenstrual Syndrome. Washington, DC.

19. Davis, A.R. \& C.L. Westhoff. 2001. Primary dysmenorrhea in adolescent girls and treatment with oral contraceptives. J. Ped. Adolesc. Gynecol. 14: 3-8.

20. Borges, L.E., R.P. Andrade, J.M. Aldrighi, et al. 2006. Effect of a combination of ethinylestradiol 30 microg and drospirenone $3 \mathrm{mg}$ on tolerance, cycle control, general well-being and fluid-related symptoms in women with premenstrual disorders requesting contraception. Contraception 74: 446-450.

21. Wyatt, K.M., P.W. Dimmock \& P.M O'Brien. 2005. Selective serotonin reuptake inhibitors for premenstrual syndrome. In The Cochrane Database of Systematic Reviews (3) Art. No: CD001396.

22. GRAY, S.H. \& S.J. EMANS. 2007. Abnormal vaginal bleeding in adolescents. Ped. Rev. 28: 175-182.

23. Albanese, A. \& N.W. Hopper. 2007. Suppression of menstruation in adolescents with severe learning disabilities. Arch. Dis. Child. 92: 629-32.

24. Bonnar, J. \& B.L. Sheppard. 1996. Treatment of menorrhagia during menstruation: randomised controlled trial of ethamsylate, mefenamic acid, and tranexamic acid. BMJ 313: $579-582$.

25. Edelman, A.B., M.F. Gallo, J.T. Jensen, et al. 2005. Continuous or extended cycle vs. cyclic use of combined oral contraceptives for contraception. In Cochrane Database Syst. Rev. CD004695.

26. Sucato, G.S. \& K.L. Gerschultz. 2005. Extended cycle hormonal contraception in adolescents. Curr. Opin. Obstet. Gynecol. 17: 461-465.
27. Rousseau, M.C. \& B. Gulllotel. 2001. Risk factors for deep venous thrombosis in tetraparesic mentally retarded patients. Brain Injury 15: 1041-1044.

28. Lohiya, G.S., F.M. Crinella, L. Tan-Figueroa \& S. Go. 2005. Deep vein thrombosis in a tetraparesic patient with mental retardation: case report and review of the literature. Brain Injury 19: 739-742.

29. Schreijer, A.J., S.C. Cannegieter, J.C. Meijers, et al. 2006. Activation of coagulation system during air travel: a crossover study. Lancet 367: 832-838.

30. TAnis, B.G. \& F.R. RosendaAl. 2003. Venous and arterial thrombosis during oral contraceptive use: risks and risk factors. Semin. Vasc. Med. 3: 69-84.

31. SAvelli, S.L., B.A. Kerlin, M.A. SPRINGER, et al. 2006. Recommendations for screening for thrombophilic tendencies in teenage females prior to contraceptive initiation. J. Ped. Adolesc. Gynecol. 19: 313-316.

32. Cole, J.A., H. Norman, M. Doherty, et al. 2007. Venous thromboembolism, myocardial infarction, and stroke among transdermal contraceptive system users. Obstet. Gynecol. 109: 339-346.

33. DiVasta, A.D. \& G.M. Gordon. 2006. Bone health in adolescents. Adolesc. Med. Clin. 17: 639-652.

34. Understanding the needs of women with epilepsy or risk OC failure. 1998. In Contraceptive Technol. Update 19: $109-1011$.

35. Arias, R.D., J.K. Jain, C. Brucker, et al. 2006. Changes in bleeding patterns with depot medroxyprogesterone acetate subcutaneous injection $104 \mathrm{mg}$. Contraception 74: 234-238.

36. Westhoff, C., J.K. Jain, I. Milsom, et al. 2007. Changes in weight with depot medroxyprogesterone acetate subcutaneous injection $104 \mathrm{mg} / 0.65 \mathrm{~mL}$. Contraception 75: 261-267.

37. Bonny, A., K. Camlin, R. Harvey, et al. 2003. Prospective analysis of weight changes in adolescent females initiating depot medroxyprogesterone acetate (DMPA), oral contraceptive pills, or no hormonal method. J. Adolesc. Health 32: 134.

38. Gromer, B.A., M. Stager, A. Bonny et al. 2004. Depot medroxyprogesterone acetate, oral contraceptives and bone mineral density in a cohort of adolescent girls. J. Adolesc. Health 35: 434-441.

39. Lopez, L.M., D.A. Grimes, K.F. Schulz, et al. 2006. Steroidal contraceptives: effect on bone fractures in women. In Cochrane Database of Systematic Reviews. (4):CD006033.

40. King, W., R. Levin, R. Schmidt, et al. 2003. Prevalence of reduced bone mass in children and adults with spastic quadriplegia. Dev. Med. Child. Neurol. 45: 12 16.

41. Henderson, R.C., R.K. Lark, M.J. Gurka, et al. 2002. Bone density and metabolism in children and adolescents with moderate to severe cerebral palsy. Pediatrics 110: 1-10.

42. Watson, K.G., M.J. Lentz \& K.C. Cain. 2006. Associations between fracture incidence and use of depot medroxyprogesterone acetate and anti-epileptic drugs in women with developmental disabilities. Womens' Health Issues 16: $346-352$. 
43. Schrager, S., C. Kloss \& A.W. Ju. 2007. Prevalence of fractures in women with intellectual disabilities: a chart review. J. Intellect. Disabil. Res. 51: 253259.

44. Pluskiewicz, W., B. Drozdzowska, A. Lyssek-Boro, et al. 2006. Densitometric and quantitative ultrasound measurements and laboratory investigations in wheelchair-bound patients. J. Clin. Densitom. 9: 78-83.

45. Funk, S., M.M. Miller, D.R. Mishell, JR., et al. 2005. The Implanon US Study Group. Safety and efficacy of Implanon, a single-rod implantable contraceptive containing etonogestrel. Contraception 71: 319-326.

46. Hidalgo, M., L. Bahamondes, M. Perrotti, et al. 2002. Bleeding patterns and clinical performance of the levonorgestrel-releasing intrauterine system (Mirena) up to two years. Contraception 65: 129 132.

47. Suhonen, S., M. Haukkamaa, T. Jakobsson \& I. Rauramo. 2004. Clinical performance of a levonorgestrel-releasing intrauterine system and oral contraceptives in young nulliparous women: a comparative study. Contraception 69: 407-412.

48. Paransky, O.I. \& R.K. Zurawin. 2003. Management of menstrual problems and contraception in adolescents with mental retardation: a medical, legal, and ethical review with new suggested guidelines.J. Pediat. Adolesc. Gynecol. 16: $223-235$.

49. American College of Obstetrics and Gynecology. 1999. Sterilization of women, including those with mental disabilities. In ACOG Committee Opinion 216. Washington, DC. 